Supporting information

\title{
Colorimetric Ionic Organohydrogels Mimicking Human Skin for Mechanical Stimuli Sensing and Injury Visualization
}

Wenlian Qiu, ${ }^{a, b}$ Changgeng Zhang, ${ }^{a}$ Guoqing Chen, ${ }^{a}$ He Zhu, ${ }^{a}$ Qi Zhang, ${ }^{* a}$ Shiping Zhu $* a$

a School of Science and Engineering, The Chinese University of Hong Kong, Shenzhen, Guangdong 518172, P. R. China

${ }^{\mathrm{b}}$ University of Science and Technology of China, Hefei, Anhui 230026, P. R. China

Email: qizhang@cuhk.edu.cn; shipingzhu@cuhk.edu.cn 


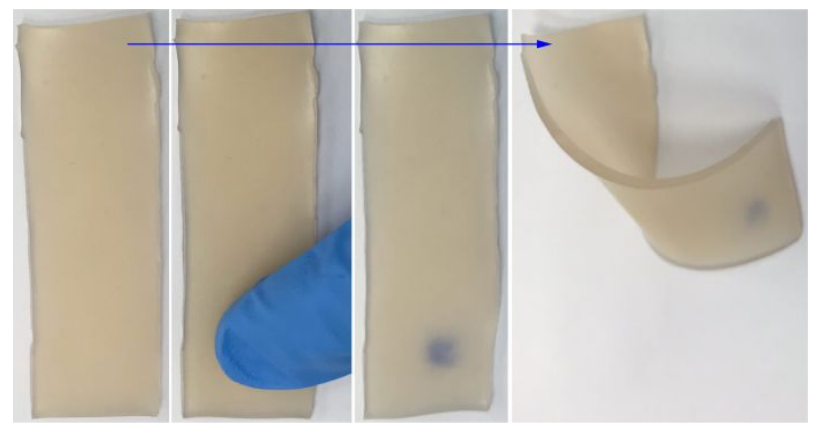

Figure S1. Photos of organohydrogel before and after pressed hard by an index finger, showing a color change but no solvent trace on the paper underneath.

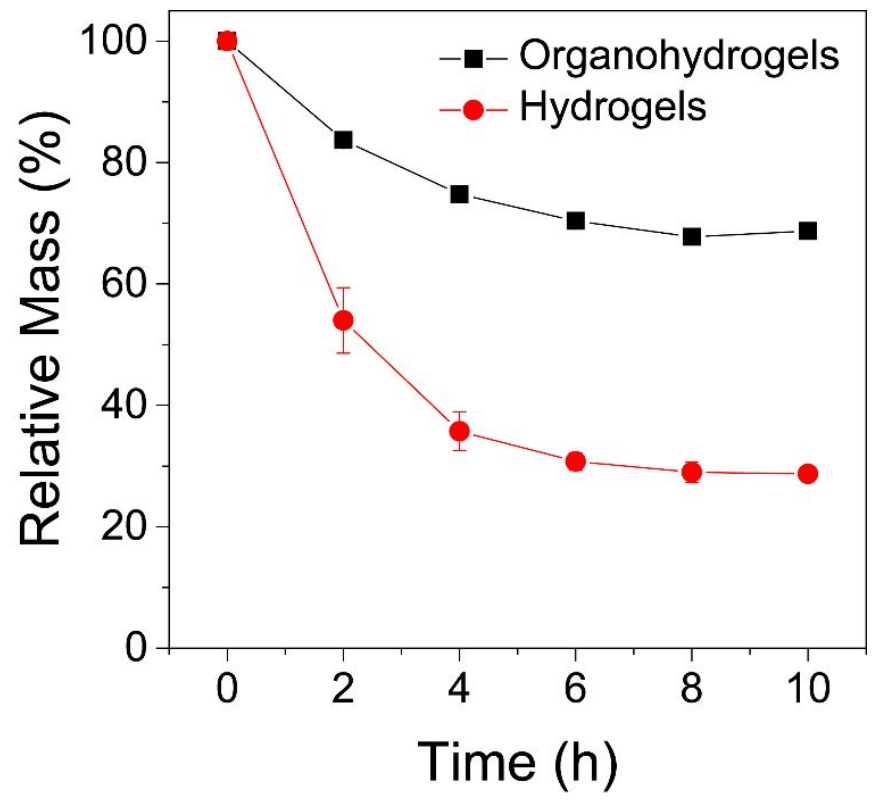

Figure S2. Solvent loss of the ionic gels under exposure to air at $30 \%$ relative humidity. 

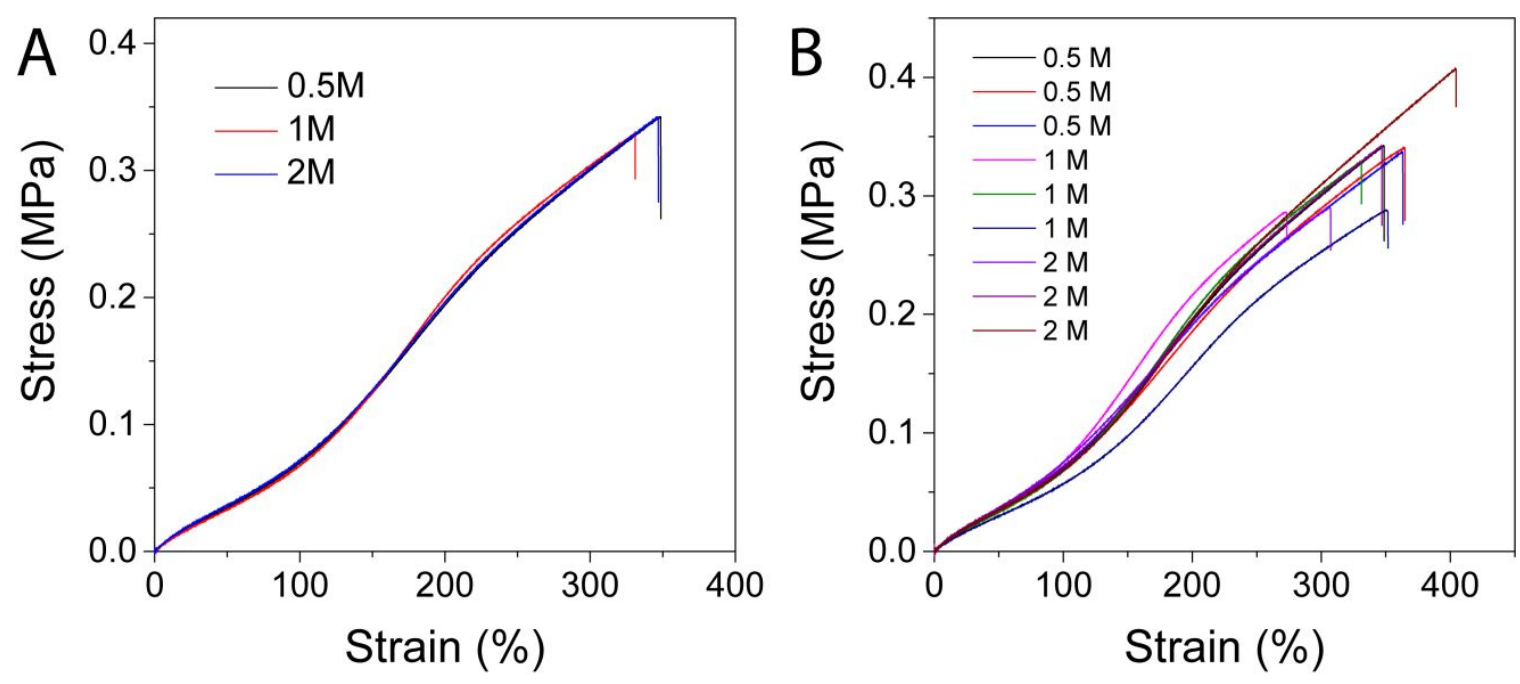

Figure S3. (A) Representative stress-strain curves and (B) more stress-strain curves of the ionic gels varying $\mathrm{NaCl}$ concentrations.
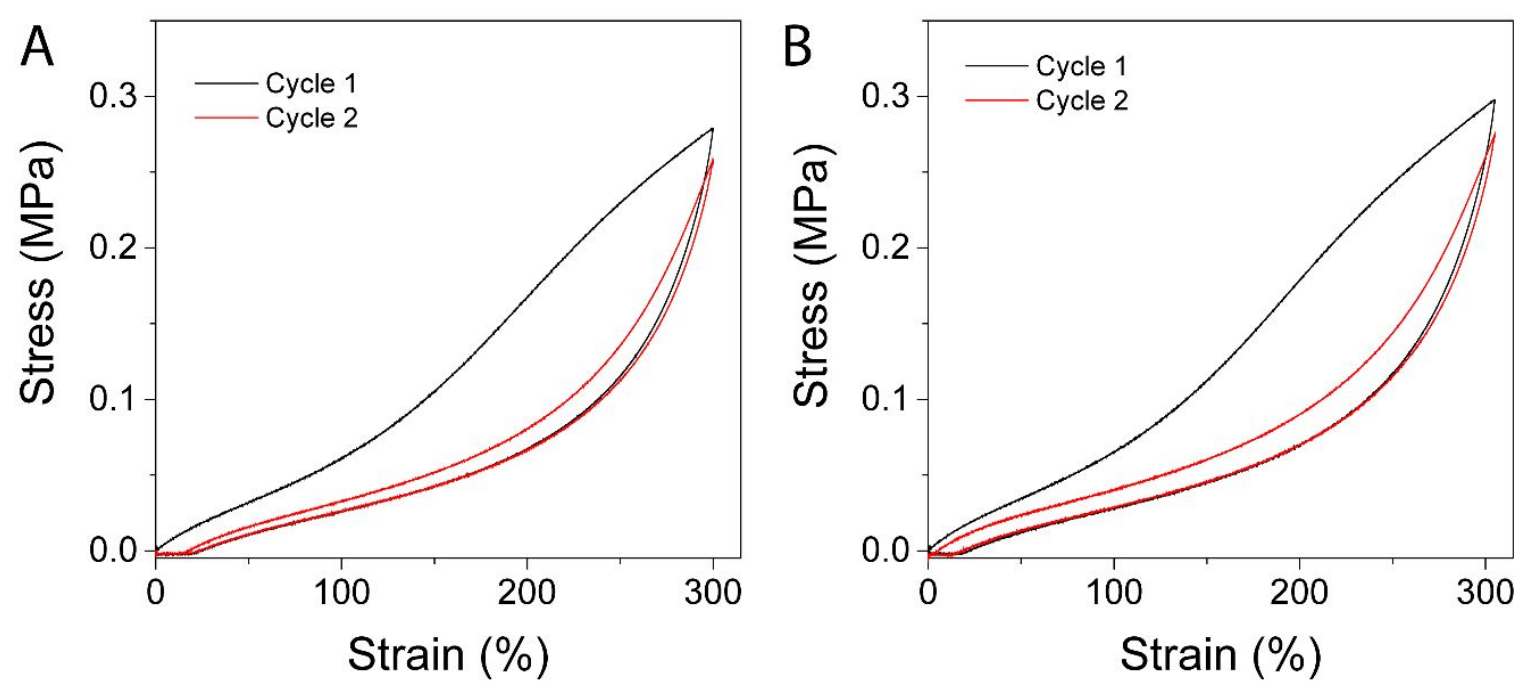

Figure S4. Cyclic stretching-releasing stress-strain curves (A) without rest time between the cycles and (B) with 30 min rest time between the cycles. 


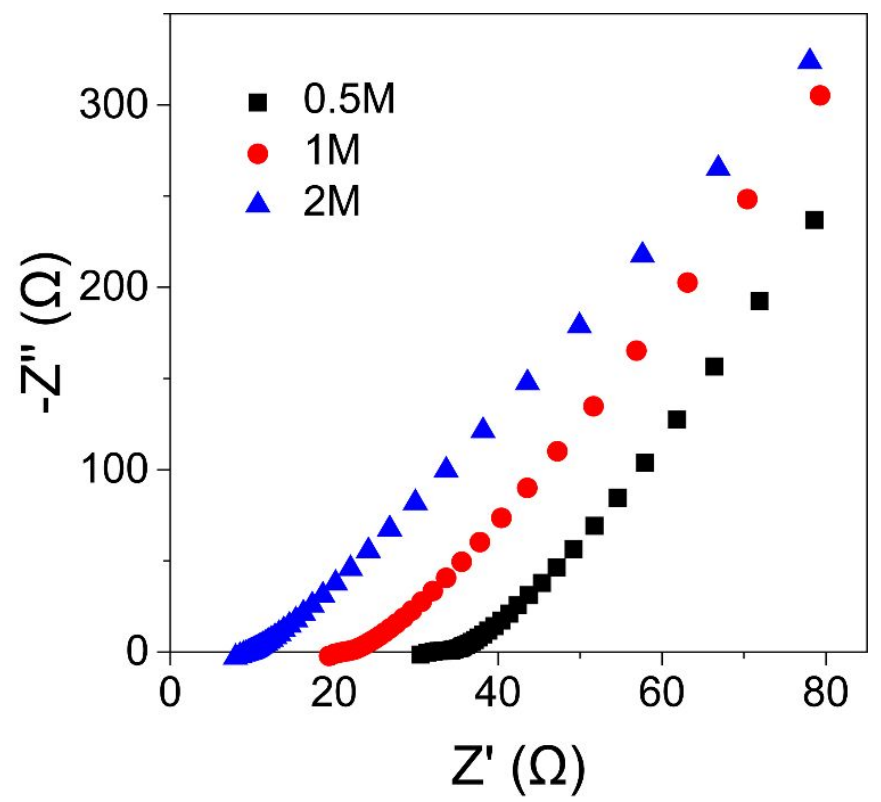

Figure S5. Representative Nyquist plots for the organohydrogles with various $\mathrm{NaCl}$ concentrations in the aqueous phase.

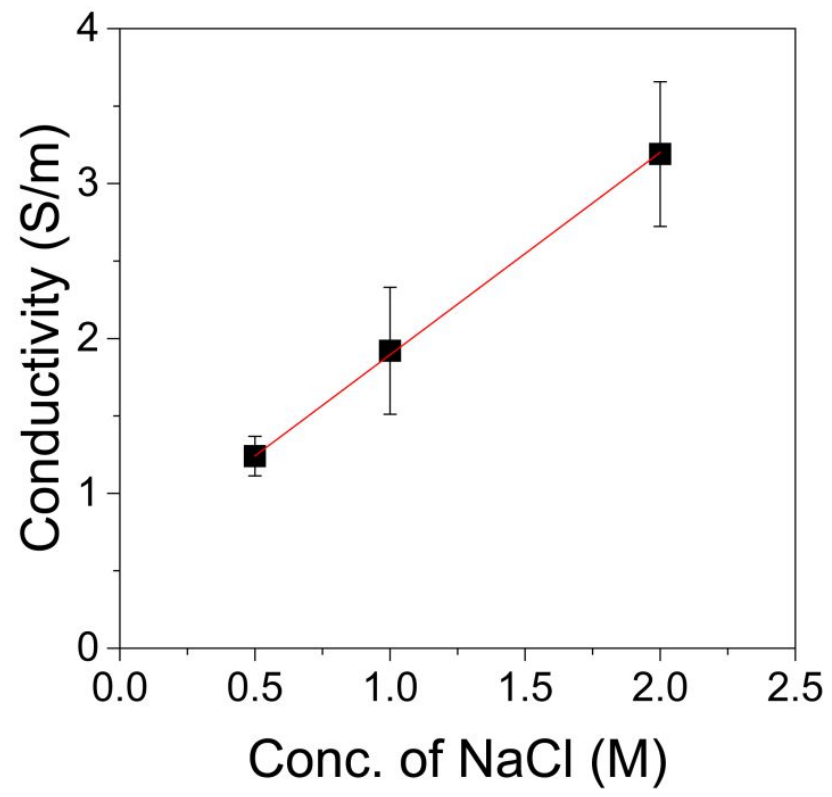

Figure S6. Relation between conductivity and $\mathrm{NaCl}$ concentration in the organohydrogels. 


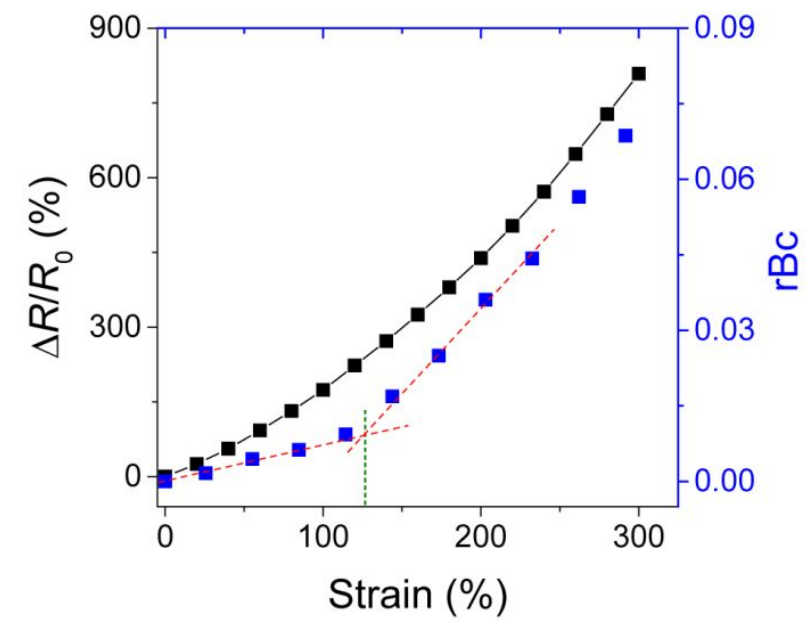

Figure S7. More plots for changes of relative resistance and blue color intensity ratio as a function of tensile strain.

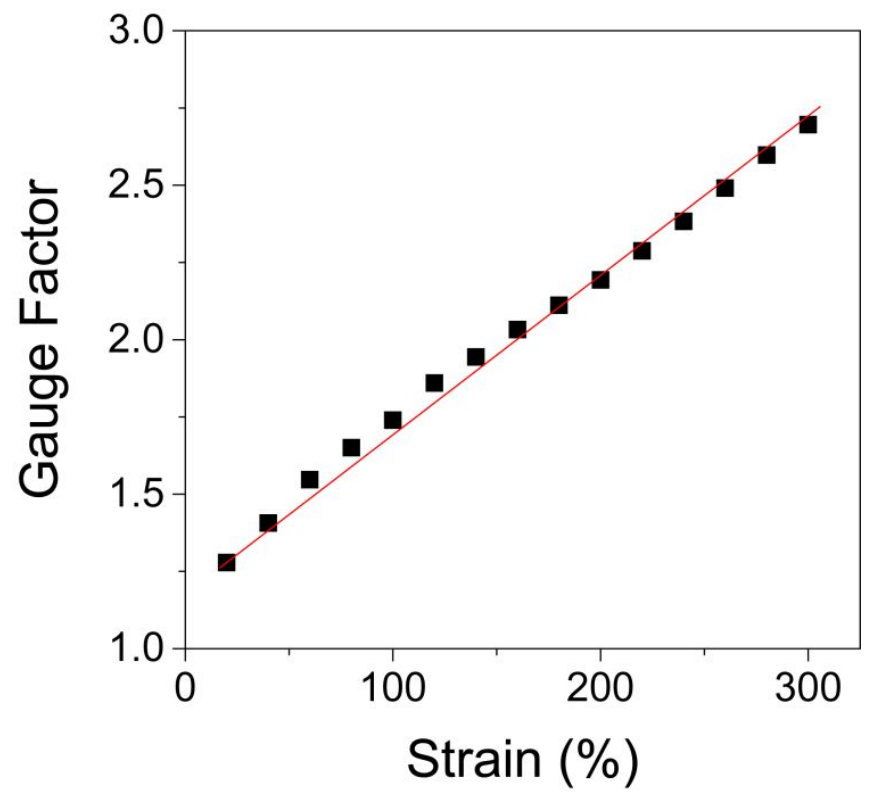

Figure S8. Gauge factor as a function of tensile strain. 

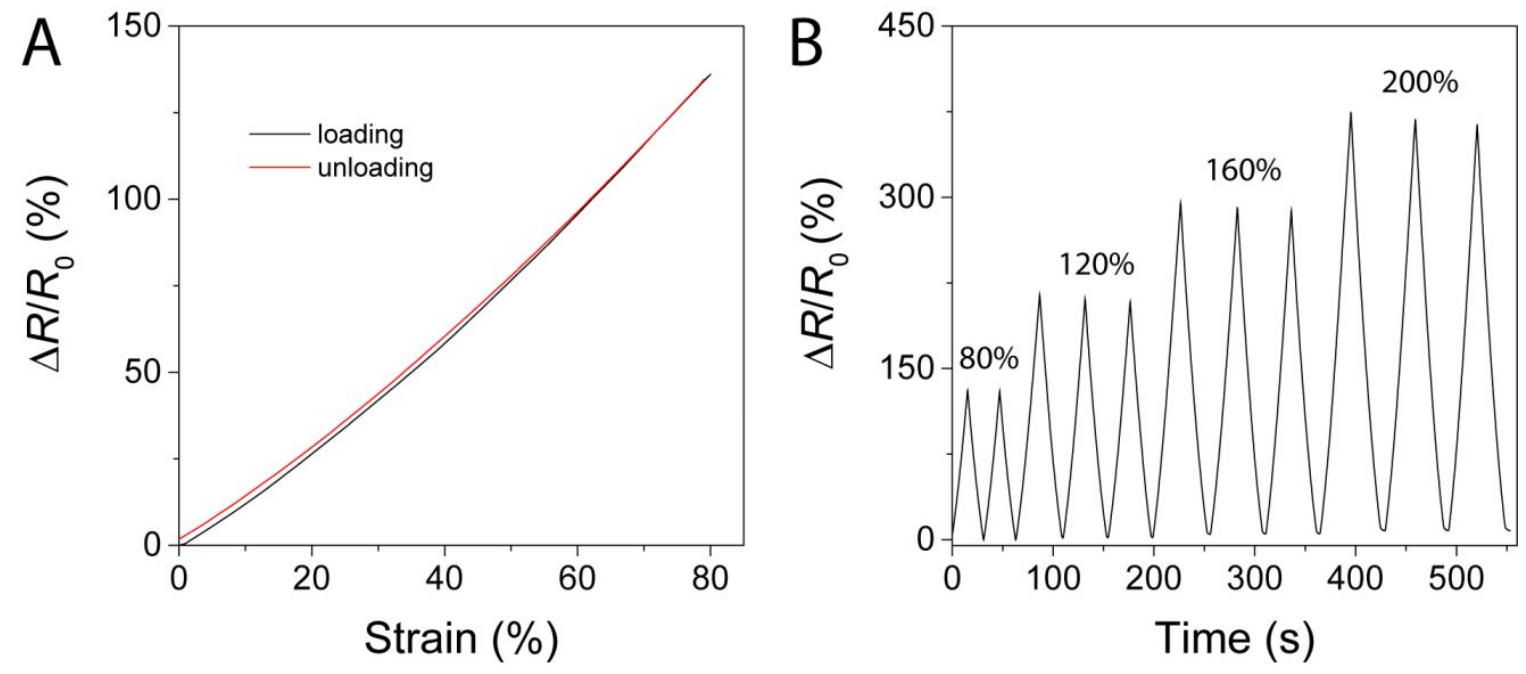

Figure S9. Relative resistance changes (A) during a stretching-releasing cycle, and (B) when repeating stretching-releasing at different strain levels.

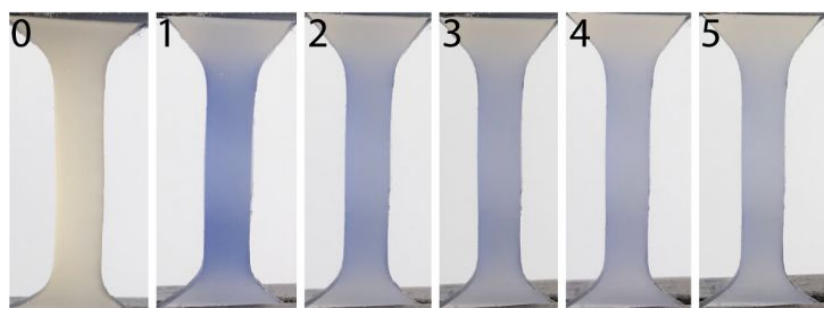

Figure S10. Images of the organohydrogel released from 300\% strain over multiple stretchingreleasing cycles, with color totally fading before the next cycle.

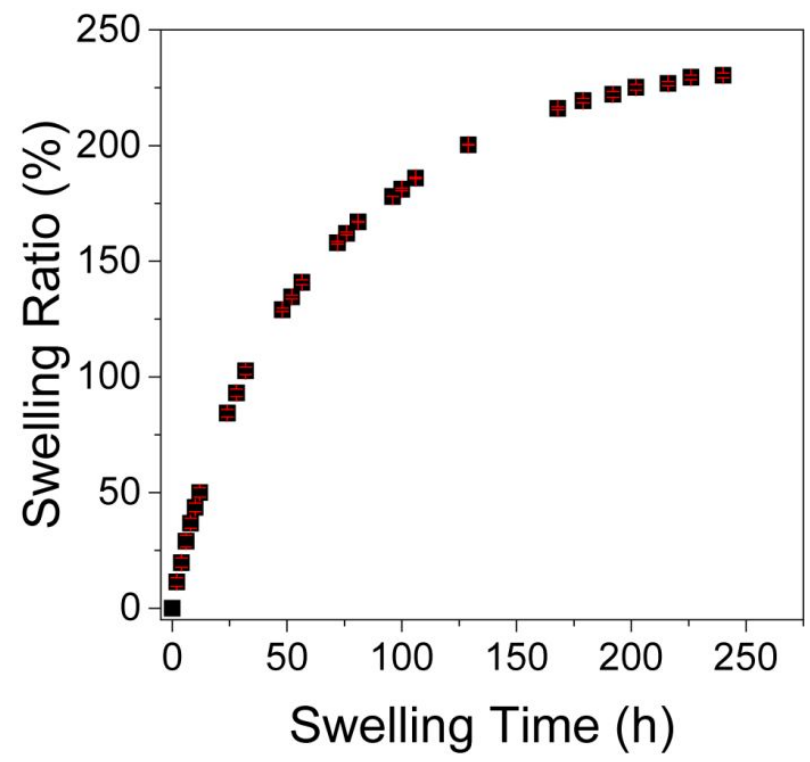

Figure S11. Swelling kinetics of cylindrical samples. Error bar is in red. 


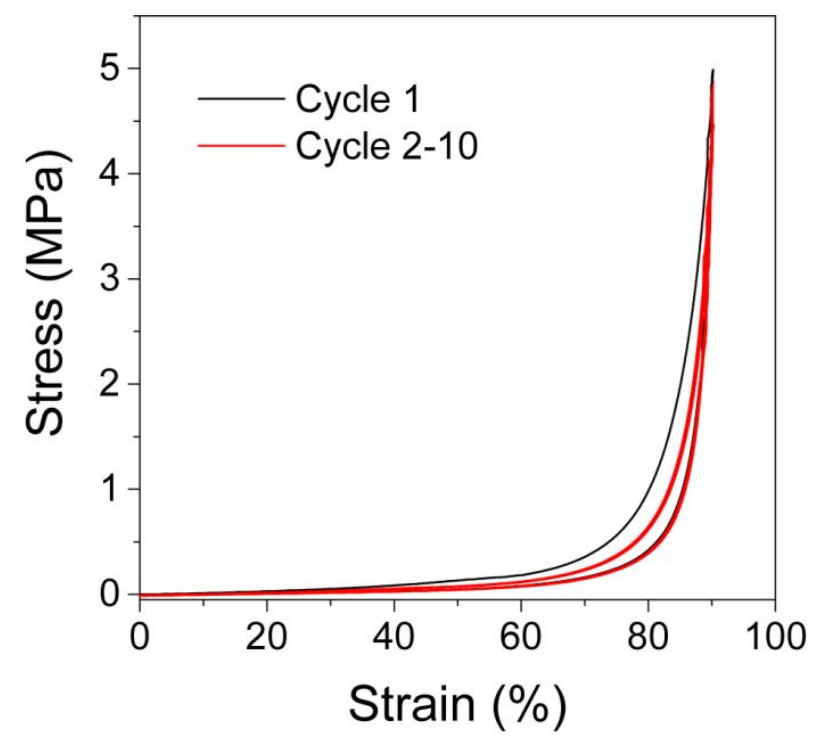

Figure S12. Stress-strain curves of cylindrical specimen under cyclic compression at $90 \%$ strain.
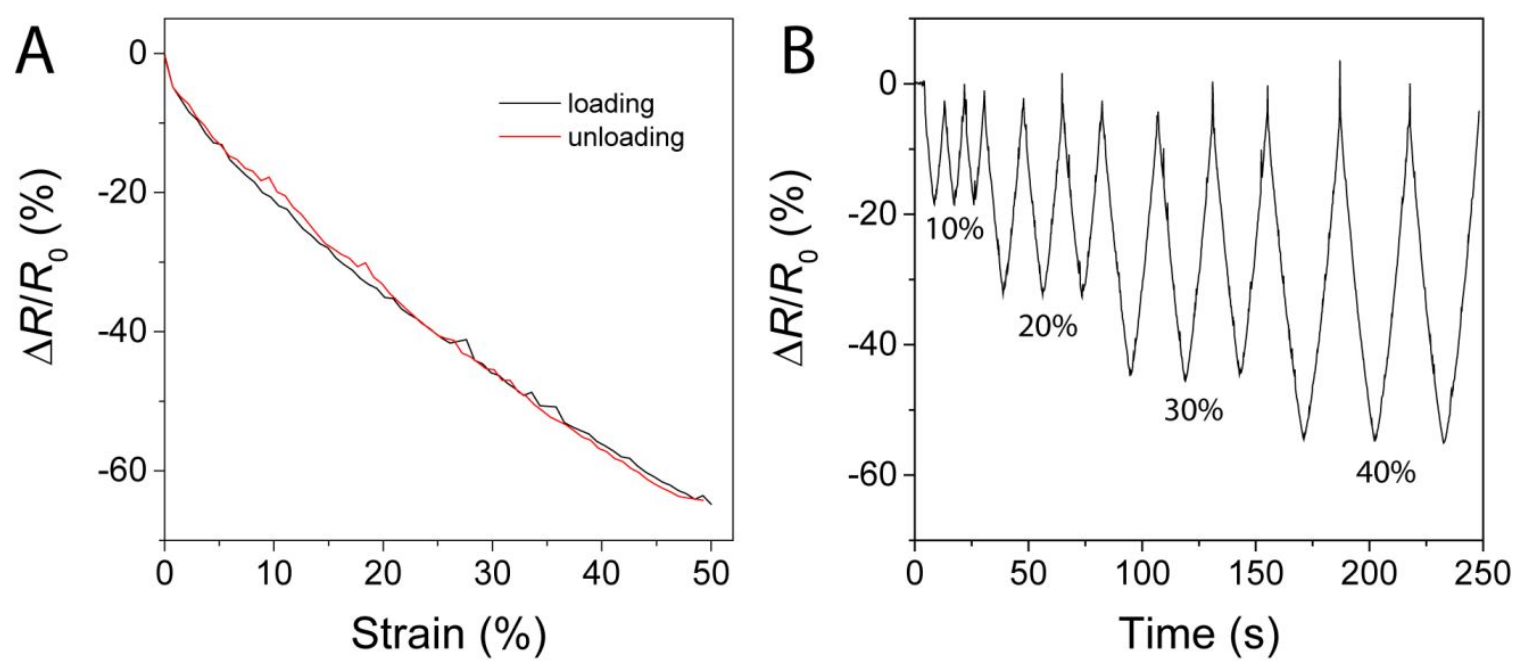

Figure S13. Relative resistance changes (A) during a compressing-releasing cycle, and (B) when repeating compressing-releasing at different strain levels. 

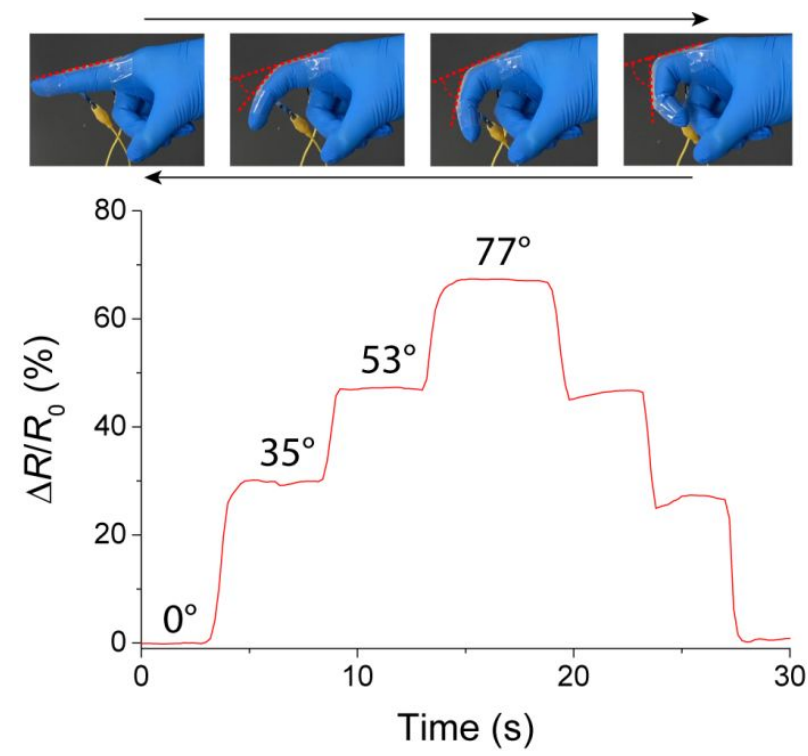

Figure S14. Relative resistance changes of the ionic skin attached on an index finger bending to different angles and then straightening gradually.
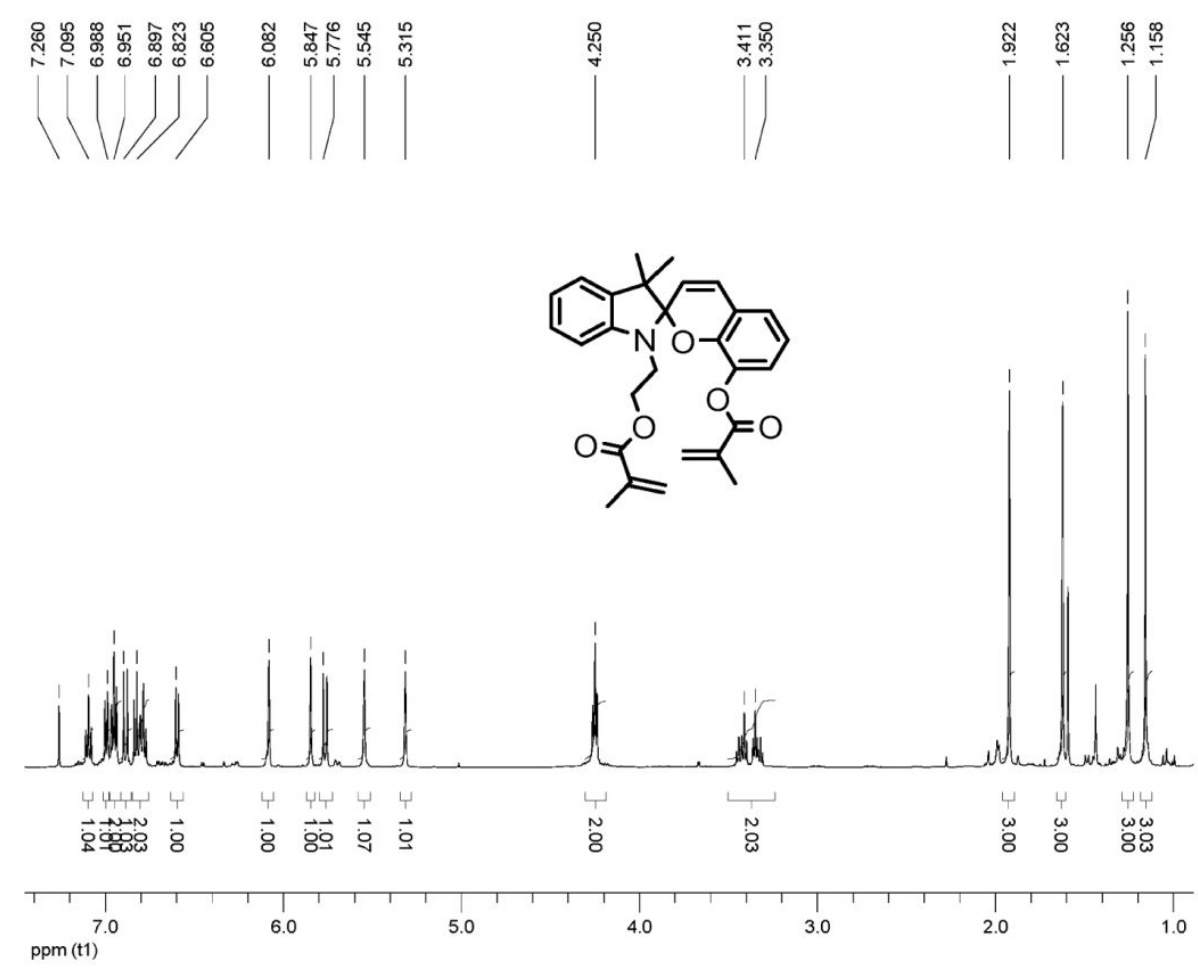

Figure S15. ${ }^{1} \mathrm{H}$ NMR spectrum of the synthesized SPMA $\left(500 \mathrm{MHz}, \mathrm{CDCl}_{3}\right)$. 